\title{
Singular Periodic Impulse Problems
}

\author{
Zdeněk Halas and Milan Tvrdý
}

October 15, 2007

\begin{abstract}
Existence principle for the impulsive periodic boundary value problem $u^{\prime \prime}+c u^{\prime}=g(x)+e(t), u\left(t_{i}+\right)=u\left(t_{i}\right)+J_{i}\left(u, u^{\prime}\right), u^{\prime}\left(t_{i}+\right)=u^{\prime}\left(t_{i}\right)+M_{i}\left(u, u^{\prime}\right), i=1, \ldots, m$, $u(0)=u(T), u^{\prime}(0)=u^{\prime}(T)$ is established, where $g \in C(0, \infty)$ can have a strong singularity at the origin. Furthermore, we assume that $0<t_{1}<\ldots<t_{m}<T, \quad e \in L_{1}[0, T]$, $c \in \mathbb{R}$ and $J_{i}, M_{i}, i=1,2, \ldots, m$, are continuous mappings of $G[0, T] \times G[0, T]$ into $\mathbb{R}$, where $G[0, T]$ denotes the space of functions regulated on $[0, T]$.

The principle is based on an averaging procedure similar to that introduced by Manásevich and Mawhin for singular periodic problems with $p$ - Laplacian in [11].

Mathematics Subject Classification 2000. 34B37, 34B15, 34C25
\end{abstract}

Keywords. impulses, periodic solutions, topological degree

\section{Preliminaries}

Starting with $\mathrm{Hu}$ and Lakshmikantham [7], periodic boundary value problems for nonlinear second order impulsive differential equations of the form

$$
\begin{aligned}
& u^{\prime \prime}=f\left(t, u, u^{\prime}\right), \\
& \left\{\begin{array}{l}
u\left(t_{i}+\right)=u\left(t_{i}\right)+J_{i}\left(u, u^{\prime}\right), \\
u^{\prime}\left(t_{i}+\right)=u^{\prime}\left(t_{i}\right)+M_{i}\left(u, u^{\prime}\right), \quad i=1,2, \ldots, m,
\end{array}\right. \\
& u(0)=u(T), \quad u^{\prime}(0)=u^{\prime}(T)
\end{aligned}
$$

have been studied by many authors. Usually it is assumed that the function $f:[0, T] \times \mathbb{R}^{2} \rightarrow \mathbb{R}$ fulfils the Carathéodory conditions,

$0<t_{1}<t_{2}<\ldots<t_{m}<T$ are fixed points of the interval $[0, T]$ 
and $J_{i}, M_{i}: \mathbb{R}^{2} \rightarrow \mathbb{R}, \quad i=1,2, \ldots, m$, are continuous functions. A rather representative (however not complete) list of related papers is given in references. In particular, in [2], [3], [5], [9], [10] existence results in terms of lower/upper functions obtained by the monotone iterative method can be found. All of these results impose monotonicity of the impulse functions and existence of an associated pair of well-ordered lower/upper functions. The papers [4] and [30] are based on the method of bound sets, however the effective criteria contained therein correspond to the situation when there is a well-ordered pair of constant lower and upper functions. Existence results which apply also to the case when a pair of lower and upper functions which need not be well-ordered is assumed were provided only by Rachůnková and Tvrdý, see [18], [20]-[22]. Analogous results for impulsive problems with quasilinear differential operator were delivered by Rachůnková and Tvrdý in [23]-[25]. When no impulses are acting, periodic problems with singularities have been treated by many authors. For rather representative overview and references, see e.g. [15] or [16]. To our knowledge, up to now singular periodic impulsive problems have not been treated. For singular Dirichlet impulsive problems we refer to the papers by Rachůnková [14], Rachůnková and Tomeček [17] and Lee and Liu [8].

In this paper we establish an existence principle suitable for solving singular impulsive periodic problems.

1.1. Notation. Throughout the paper we keep the following notation and conventions: for a real valued function $u$ defined a.e. on $[0, T]$, we put

$$
\|u\|_{\infty}=\sup _{\operatorname{ess}_{t \in[0, T]}}|u(t)| \quad \text { and } \quad\|u\|_{1}=\int_{0}^{T}|u(s)| \mathrm{d} s .
$$

For a given interval $J \subset \mathbb{R}$, by $C(J)$ we denote the set of real valued functions which are continuous on $J$. Furthermore, $C^{1}(J)$ is the set of functions having continuous first derivatives on $J$ and $L_{1}(J)$ is the set of functions which are Lebesgue integrable on $J$.

Any function $x:[0, T] \rightarrow \mathbb{R}$ which possesses finite limits

$$
x(t+)=\lim _{\tau \rightarrow t+} x(\tau) \quad \text { and } \quad x(s-)=\lim _{\tau \rightarrow s-} x(\tau)
$$

for all $t \in[0, T)$ and $s \in(0, T]$ is said to be regulated on $[0, T]$. The linear space of functions regulated on $[0, T]$ is denoted by $G[0, T]$. It is well known 
that $G[0, T]$ is a Banach space with respect to the norm $x \in G[0, T] \rightarrow\|x\|_{\infty}$ (cf. [6, Theorem I.3.6]).

Let $m \in \mathbb{N}$ and let $0=t_{0}<t_{1}<t_{2}<\cdots<t_{m}<t_{m+1}=T \quad$ be a division of the interval $[0, T]$. We denote $D=\left\{t_{1}, t_{2}, \ldots, t_{m}\right\}$ and define $C_{D}^{1}[0, T]$ as the set of functions $u:[0, T] \rightarrow \mathbb{R}$ such that

$$
u(t)= \begin{cases}u_{[0]}(t) & \text { if } t \in\left[0, t_{1}\right], \\ u_{[1]}(t) & \text { if } t \in\left(t_{1}, t_{2}\right], \\ \vdots & \vdots \\ u_{[m]}(t) & \text { if } t \in\left(t_{m}, T\right],\end{cases}
$$

where $u_{[i]} \in C^{1}\left[t_{i}, t_{i+1}\right]$ for $i=0,1, \ldots, m$. In particular, if $u \in C_{D}^{1}[0, T]$, then $u^{\prime}$ possesses finite one-sided limits

$$
u^{\prime}(t-):=\lim _{\tau \rightarrow t-} u(\tau) \text { and } \quad u^{\prime}(s+):=\lim _{\tau \rightarrow s+} u(\tau)
$$

for each $t \in(0, T]$ and $s \in[0, T)$. Moreover, $u^{\prime}(t-)=u^{\prime}(t)$ for all $t \in$ $(0, T]$ and $u^{\prime}(0+)=u^{\prime}(0)$. For $u \in C_{D}^{1}[0, T]$ we put

$$
\|u\|_{D}=\|u\|_{\infty}+\left\|u^{\prime}\right\|_{\infty} .
$$

Then $C_{D}^{1}[0, T]$ becomes a Banach space when endowed with the norm $\|\cdot\|_{D}$. Furthermore, by $A C_{D}^{1}[0, T]$ we denote the set of functions $u \in C_{D}^{1}[0, T]$ having first derivatives absolutely continuous on each subinterval $\left(t_{i}, t_{i+1}\right)$, $i=1,2, \ldots, m+1$.

We say that $f:[0, T] \times \mathbb{R}^{2} \mapsto \mathbb{R}$ satisfies the Carathéodory conditions on $[0, T] \times \mathbb{R}^{2}$ if (i) for each $x \in \mathbb{R}$ and $y \in \mathbb{R}$ the function $f(., x, y)$ is measurable on $[0, T]$; (ii) for almost every $t \in[0, T]$ the function $f(t, .,$. is continuous on $\mathbb{R}^{2}$; (iii) for each compact set $K \subset \mathbb{R}^{2}$ there is a function $m_{K}(t) \in L[0, T]$ such that $|f(t, x, y)| \leq m_{K}(t)$ holds for a.e. $t \in[0, T]$ and all $(x, y) \in K$. The set of functions satisfying the Carathéodory conditions on $[0, T] \times \mathbb{R}^{2}$ is denoted by $\operatorname{Car}\left([0, T] \times \mathbb{R}^{2}\right)$.

Given a subset $\Omega$ of a Banach space $X$, its closure is denoted by $\bar{\Omega}$. Finally, we will write $\bar{e}$ instead of $\frac{1}{T} \int_{0}^{T} e(s) \mathrm{d} s$ and $\Delta^{+} u(t)$ instead of $u(t+)-u(t)$. 
If $f \in \operatorname{Car}\left([0, T] \times \mathbb{R}^{2}\right)$, problem (1.1)-(1.3) is said to be regular and a function $u \in A C_{D}^{1}[0, T]$ is its solutions if

$$
u^{\prime \prime}(t)=f\left(t, u(t), u^{\prime}(t)\right) \text { holds for a.e. } t \in[0, T]
$$

and conditions (1.2) and (1.3) are satisfied. If $f \notin \operatorname{Car}\left([0, T] \times \mathbb{R}^{2}\right)$, problem (1.1)-(1.3) is said to be singular.

In this paper we will deal with rather simplified, however the most typical, case of the singular problem with

$$
f(t, x, y)=c y+g(x)+e(t) \text { for } x \in(0, \infty), y \in \mathbb{R} \text { and a.e. } t \in[0, T],
$$
where

$$
c \in \mathbb{R}, \quad g \in C(0, \infty), \quad e \in L_{1}[0, T] .
$$

1.2. Definition. A function $u \in A C_{D}^{1}[0, T]$ is called a solution of problem

$$
u^{\prime \prime}+c u^{\prime}=g(u)+e(t), \quad(1.2),(1.3)
$$

if $u>0$ a.e. on $[0, T]$,

$$
u^{\prime \prime}(t)+c u^{\prime}(t)=g(u(t))+e(t) \quad \text { for a.e. } t \in[0, T],
$$

and conditions (1.2) and (1.3) are satisfied.

\section{Green's functions and operator represen- tations for impulsive two-point boundary value problems}

For our purposes an appropriate choice of the operator representation of (1.1)-(1.3) is important. To this aim, let us consider the following impulsive problem with nonlinear two-point boundary conditions

$$
\begin{aligned}
& u^{\prime \prime}+a_{2}(t) u^{\prime}+a_{1}(t) u=f\left(t, u, u^{\prime}\right) \text { a.e. on }[0, T] \\
& \Delta^{+} u\left(t_{i}\right)=J_{i}\left(u, u^{\prime}\right), \quad \Delta^{+} u^{\prime}\left(t_{i}\right)=M_{i}\left(u, u^{\prime}\right), \quad i=1,2, \ldots, m \\
& P\left(\begin{array}{c}
u(0) \\
u^{\prime}(0)
\end{array}\right)+Q\left(\begin{array}{c}
u(T) \\
u^{\prime}(T)
\end{array}\right)=R\left(u, u^{\prime}\right),
\end{aligned}
$$


and its linearized version

$$
\begin{aligned}
& u^{\prime \prime}+a_{2}(t) u^{\prime}+a_{1}(t) u=h(t) \text { a.e. on }[0, T], \\
& \Delta^{+} u\left(t_{i}\right)=d_{i}, \quad \Delta^{+} u^{\prime}\left(t_{i}\right)=d_{i}^{\prime}, \quad i=1,2, \ldots, m, \\
& P\left(\begin{array}{c}
u(0) \\
u^{\prime}(0)
\end{array}\right)+Q\left(\begin{array}{c}
u(T) \\
u^{\prime}(T)
\end{array}\right)=\delta,
\end{aligned}
$$

where

$$
\left\{\begin{array}{l}
a_{1}, h \in L[0, T], a_{2} \in C[0, T], f \in C a r\left([0, T] \times \mathbb{R}^{2}\right), \\
J_{i} \text { and } M_{i}: G[0, T] \times G[0, T] \rightarrow \mathbb{R}, i=1,2, \ldots, m, \\
\quad \text { are continuous mappings, } \\
\delta \in \mathbb{R}^{2}, d_{i}, d_{i}^{\prime} \in \mathbb{R}, i=1,2, \ldots, m, \\
P, Q \text { are real } 2 \times 2-\text { matrices, } \operatorname{rank}(P, Q)=2, \\
R: G[0, T] \times G[0, T] \rightarrow \mathbb{R}^{2} \text { is a continuous mapping. }
\end{array}\right.
$$

Solutions of problems (2.1)-(2.3) and (2.4)-(2.6) are defined in a natural way quite analogously to the above mentioned definition of regular periodic problems. Problem (2.4)-(2.6) is equivalent to the two-point problem for a special case of generalized linear differential systems of the form

$$
\begin{aligned}
& x(t)-x(0)-\int_{0}^{t} A(s) x(s) \mathrm{d} s=b(t)-b(0) \quad \text { on }[0, T], \\
& P x(0)+Q x(T)=\delta,
\end{aligned}
$$

where

$$
\begin{aligned}
& x(t)=\left(\begin{array}{l}
x_{1}(t) \\
x_{2}(t)
\end{array}\right)=\left(\begin{array}{c}
u(t) \\
u^{\prime}(t)
\end{array}\right), \quad A(t)=\left(\begin{array}{cc}
0 & 1 \\
-a_{1}(t) & -a_{2}(t)
\end{array}\right), \\
& b(t)=\int_{0}^{t}\left(\begin{array}{c}
0 \\
h(s)
\end{array}\right) \mathrm{d} s+\sum_{i=1}^{m}\left(\begin{array}{l}
d_{i} \\
d_{i}^{\prime}
\end{array}\right) \chi_{\left(t_{i}, T\right]}(t), \quad t \in[0, T],
\end{aligned}
$$

and $\chi_{\left(t_{i}, T\right]}(t)=1$ if $t \in\left(t_{i}, T\right], \quad \chi_{\left(t_{i}, T\right]}(t)=0$ otherwise. Solutions of $(2.8),(2.9)$ are 2 -vector functions of bounded variation on $[0, T]$ satisfying 
the two-point condition (2.9) and fulfilling the integral equation (2.8) for all $t \in[0, T]$, cf. e.g. [28]. Assume that the homogeneous problem

$$
u^{\prime \prime}+a_{2}(t) u^{\prime}+a_{1}(t) u=0, \quad P\left(\begin{array}{c}
u(0) \\
u^{\prime}(0)
\end{array}\right)+Q\left(\begin{array}{c}
u(T) \\
u^{\prime}(T)
\end{array}\right)=0
$$

has only the trivial solution. Then, obviously, the problem

$$
x^{\prime}-A(t) x=0, \quad P x(0)+Q x(T)=0
$$

has also only the trivial solution. In view of [29, Theorems 4.2 and 4.3] (see also [27, Theorem 4.1]), problem (2.8), (2.9) has a unique solution $x$ and it is given by

$$
x(t)=\int_{0}^{T} \Gamma(t, s) \mathrm{d}[b(s)]+x_{0}(t), \quad t \in[0, T],
$$

where $x_{0}$ is the uniquely determined solution of

$$
x^{\prime}-A(t) x=0, P x(0)+Q x(T)=\delta
$$

and

$$
\Gamma(t, s)=\left(\gamma_{i, j}(t, s)\right)_{i, j=1,2}
$$

is Green's matrix for (2.12). Recall that, for each $s \in(0, T)$, the matrix function $t \rightarrow \Gamma(t, s)$ is absolutely continuous on $[0, T] \backslash\{s\}$ and

$$
\begin{aligned}
& \frac{\partial}{\partial t} \Gamma(t, s)-A(t) \Gamma(t, s)=0 \quad \text { for a.e. } t \in[0, T] \\
& P \Gamma(0, s)+Q \Gamma(T, s)=0 \\
& \Gamma(t+, t)-\Gamma(t-, t)=I
\end{aligned}
$$

where $I$ stands for the identity $2 \times 2$-matrix. In particular, the component $\gamma_{1,2}$ of $\Gamma$ is absolutely continuous on $[0, T]$ for each $s \in(0, T)$ and

$$
\frac{\partial}{\partial t} \gamma_{1,2}(t, s)=\gamma_{2,2}(t, s) \text { for a.e. } t \in[0, T] .
$$

Denote $G(t, s)=\gamma_{1,2}(t, s)$. Then $G(t, s)$ is Green's function of (2.11). Furthermore, we have

$$
\frac{\partial}{\partial s} \Gamma(t, s)=-\Gamma(t, s) A(s) \quad \text { for all } t \in(0, T) \text { and a.e. } s \in[0, T] .
$$


In particular,

$$
\gamma_{1,1}(t, s)=-\frac{\partial}{\partial s} G(t, s)+a_{1}(s) G(t, s) \quad \text { for all } t \in[0, T] \text { and a.e. } s \in[0, T] \text {. }
$$

Inserting (2.10) into (2.13) we get that, for each $h \in L[0, T], \quad c, d_{i}, d_{i}^{\prime} \in R$, $i=1,2, \ldots, m$, the unique solution $u$ of problem (2.4)-(2.6) is given by

$$
\left\{\begin{aligned}
u(t)= & u_{0}(t)+\int_{0}^{t} G(t, s) h(s) \mathrm{d} s \\
& +\sum_{i=1}^{m}\left(-\frac{\partial}{\partial s} G\left(t, t_{i}\right)+a_{1}(t) G\left(t, t_{i}\right)\right) d_{i}+\sum_{i=1}^{m} G\left(t, t_{i}\right) d_{i}^{\prime}
\end{aligned}\right.
$$

for $t \in[0, T]$,

where $u_{0}$ is the uniquely determined solution of the problem

$$
u^{\prime \prime}+a_{2}(t) u^{\prime}+a_{1}(t) u=0, \quad(2.6) .
$$

Now, choose an arbitrary $w \in C_{D}^{1}[0, T]$ and put

$$
\left\{\begin{array}{l}
h(t)=f\left(t, w(t), w^{\prime}(t)\right) \quad \text { for a.e. } t \in[0, T] \\
d_{i}=J_{i}\left(w, w^{\prime}\right), d_{i}^{\prime}=M_{i}\left(w, w^{\prime}\right), i=1,2, \ldots, m \\
\delta=R\left(w, w^{\prime}\right) .
\end{array}\right.
$$

Then $h \in L[0, T], \quad c, d_{i}, d_{i}^{\prime} \in \mathbb{R}, i=1,2, \ldots, m$, and there is a unique $u \in A C_{D}^{1}[0, T]$ fulfilling (2.4)-(2.6) and it is given by (2.15). Therefore, assuming, in addition, that the problem

$$
\begin{aligned}
& u^{\prime \prime}+a_{2}(t) u^{\prime}+a_{1}(t) u=0 \\
& P\left(\begin{array}{c}
u(0) \\
u^{\prime}(0)
\end{array}\right)+Q\left(\begin{array}{c}
u(T) \\
u^{\prime}(T)
\end{array}\right)=R\left(u, u^{\prime}\right)
\end{aligned}
$$


has a unique solution $u_{0}$, we conclude that $u \in C_{D}^{1}[0, T]$ is a solution to (2.1)-(2.3) if and only if

$$
\left\{\begin{aligned}
u(t)= & u_{0}(t)+\int_{0}^{t} G(t, s) f\left(s, u(s), u^{\prime}(s)\right) \mathrm{d} s \\
& +\sum_{\substack{i=1 \\
m}}^{m}\left(-\frac{\partial}{\partial s} G\left(t, t_{i}\right)+a_{1}(t) G\left(t, t_{i}\right)\right) J_{i}\left(u, u^{\prime}\right) \\
& +\sum_{i=1}^{m} G\left(t, t_{i}\right) M_{i}\left(u, u^{\prime}\right) \quad \text { for } t \in[0, T] .
\end{aligned}\right.
$$

Let us define operators $F_{1}$ and $F_{2}: C_{D}^{1}[0, T] \rightarrow C_{D}^{1}[0, T]$ by

$$
\left(F_{1} u\right)(t)=\int_{0}^{T} G(t, s) f\left(s, u(s), u^{\prime}(s)\right) \mathrm{d} s, \quad t \in[0, T]
$$

and

$$
\begin{aligned}
\left(F_{2} u\right)(t)= & u_{0}(t)+\sum_{i=1}^{m}\left(-\frac{\partial}{\partial s} G\left(t, t_{i}\right)+a_{1}(t) G\left(t, t_{i}\right)\right) J_{i}\left(u, u^{\prime}\right) \\
& +\sum_{i=1}^{m} G\left(t, t_{i}\right) M_{i}\left(u, u^{\prime}\right), \quad t \in[0, T] .
\end{aligned}
$$

The former one, $F_{1}$, is a composition of the Green type operator

$$
h \in L_{1}[0, T] \rightarrow \int_{0}^{T} G(t, s) h(s) \mathrm{d} s \in C^{1}[0, T],
$$

which is known to map equiintegrable subsets ${ }^{1}$ of $L_{1}[0, T]$ onto relatively compact subsets of $C^{1}[0, T] \subset C_{D}^{1}[0, T]$, and of the superposition operator generated by $f \in \operatorname{Car}\left([0, T] \times \mathbb{R}^{2}\right)$, which similarly to the classical setting maps bounded subsets of $C_{D}^{1}[0, T]$ to equiintegrable subsets of $L_{1}[0, T]$. Therefore, it is easy to see that $F_{1}$ is completely continuous. Furthermore, since $J_{i}, M_{i}, i=1,2, \ldots, m$, are continuous mappings, the operator $F_{2}$ is continuous as well. Having in mind that $F_{2}$ maps bounded sets onto bounded sets and its values are contained in a $(2 m+1)$-dimensional subspace $^{2}$ of $C_{D}^{1}[0, T]$, we conclude that the operators $F_{2}$ and $F=F_{1}+F_{2}$ are completely continuous as well.

So, we have the following assertion.

\footnotetext{
${ }^{1}$ i.e. sets of functions having a common integrable majorant

${ }^{2}$ i.e. spanned over the set $\left\{u_{0}, G\left(., t_{i}\right),\left(-\frac{\partial}{\partial s} G\left(., t_{i}\right)+a_{1} G\left(., t_{i}\right)\right), i=1,2, \ldots, m\right\}$
} 
2.1. Proposition. Assume (1.4) and (2.7). Furthermore, let problem (2.11) have Green's function $G(t, s)$ and let $u_{0} \in A C_{D}^{1}[0, T]$ be a uniquely defined solution of problem (2.17), (2.18). Then $u \in A C_{D}^{1}$ is a solution to (2.1)-(2.3) if and only if $u=F u$, where $F: C_{D}^{1}[0, T] \rightarrow C_{D}^{1}[0, T]$ is the completely continuous operator given by

$$
\left\{\begin{array}{l}
(F u)(t)=u_{0}(t) \\
+\int_{0}^{T} G(t, s)\left(f\left(t, u(s), u^{\prime}(s)\right)-a_{1}(s) u(s)-a_{2}(s) u^{\prime}(s)\right) \mathrm{d} s \\
+\sum_{i=1}^{m}\left(-\frac{\partial}{\partial s} G\left(t, t_{i}\right)+a_{1}(t) G\left(t, t_{i}\right)\right) J_{i}\left(u, u^{\prime}\right) \\
+\sum_{i=1}^{m} G\left(t, t_{i}\right) M_{i}\left(u, u^{\prime}\right), t \in[0, T] .
\end{array}\right.
$$

In particular, if $a_{1}(t)=a_{2}(t)=0$ on $[0, T]$,

$$
P=\left(\begin{array}{ll}
1 & 0 \\
0 & 0
\end{array}\right) \text { and } Q=\left(\begin{array}{ll}
0 & 0 \\
1 & 0
\end{array}\right)
$$

then problem (2.11) reduces to the simple Dirichlet problem

$$
u^{\prime \prime}=0, \quad u(0)=u(T)=0
$$

and its Green's function is well-known:

$$
G(t, s)= \begin{cases}\frac{s(t-T)}{T} & \text { if } 0 \leq s<t \leq T \\ \frac{t(s-T)}{T} & \text { if } 0 \leq t \leq s \leq T\end{cases}
$$

and

$$
\frac{\partial}{\partial s} G(t, s)= \begin{cases}\frac{T-t}{T} & \text { if } 0 \leq s<t \leq T \\ -\frac{t}{T} & \text { if } 0 \leq t \leq s \leq T\end{cases}
$$

Furthermore, let us notice that the periodic boundary conditions (1.3) can be reformulated as

$$
u(0)=u(T)=u(0)+u^{\prime}(0)-u^{\prime}(T),
$$


i.e., in the form (2.18), where

$$
R(u, v)=u(0)+v(0)-v(T) \quad \text { for } \quad u, v \in G[0, T] .
$$

It is easy to see that, in such a case, for any $c \in \mathbb{R}$ the only solution to (2.17), (2.18) is $u_{0}(t) \equiv c$. Therefore, we have the following corollary of Proposition 2.1:

2.2 . Corollary. Assume (1.4) and (2.7) and let the function $G(t, s)$ be given by (2.21). Then $u \in A C_{D}^{1}$ is a solution to (1.1)-(1.3) if and only if $u=F u$, where $F: C_{D}^{1}[0, T] \rightarrow C_{D}^{1}[0, T]$ is the completely continuous operator given by

$$
\left\{\begin{array}{l}
(F u)(t)=u(0)+u^{\prime}(0)-u^{\prime}(T)+\int_{0}^{T} G(t, s) f\left(t, u(s), u^{\prime}(s)\right) \mathrm{d} s \\
\quad-\sum_{i=1}^{m} \frac{\partial}{\partial s} G\left(t, t_{i}\right) J_{i}\left(u, u^{\prime}\right) \\
+\sum_{i=1}^{m} G\left(t, t_{i}\right) M_{i}\left(u, u^{\prime}\right), t \in[0, T] .
\end{array}\right.
$$

2.3. Remark. Similarly, $u \in A C_{D}^{1}$ is a solution to the impulsive Dirichlet problem (1.1), (1.2), $u(0)=u(T)=c$ if and only if $u=F_{d i r} u$, where

$$
\left\{\begin{array}{l}
\left(F_{\text {dir }} u\right)(t)=c+\int_{0}^{T} G(t, s) f\left(t, u(s), u^{\prime}(s)\right) \mathrm{d} s \\
\quad-\sum_{i=1}^{m} \frac{\partial}{\partial s} G\left(t, t_{i}\right) J_{i}\left(u, u^{\prime}\right)+\sum_{i=1}^{m} G\left(t, t_{i}\right) M_{i}\left(u, u^{\prime}\right), t \in[0, T] .
\end{array}\right.
$$

\section{Existence principle}

3.1. Theorem. Let assumptions (1.4) and (1.5) hold. Furthermore, assume that there exist $r \in(0, \infty), \quad R \in(r, \infty)$ and $R^{\prime} \in(0, \infty)$ such that

(i) $r<v<R$ on $[0, T]$ and $\left\|v^{\prime}\right\|_{\infty}<R^{\prime}$ for each $\lambda \in(0,1]$ and for 
each positive solution $v$ of the problem

$$
\begin{aligned}
& v^{\prime \prime}(t)=\lambda\left(-c v^{\prime}(t)+g(v(t))+e(t)\right) \quad \text { for a.e. } t \in[0, T], \\
& \Delta^{+} v\left(t_{i}\right)=\lambda J_{i}\left(v, v^{\prime}\right), \quad i=1,2, \ldots, m, \\
& \Delta^{+} v^{\prime}\left(t_{i}\right)=\lambda M_{i}\left(v, v^{\prime}\right), \quad i=1,2, \ldots, m, \\
& v(0)=v(T), \quad v^{\prime}(0)=v^{\prime}(T) ;
\end{aligned}
$$

(ii) $(g(x)+\bar{e}=0) \quad \Longrightarrow \quad r<x<R$;

(iii) $(g(r)+\bar{e})(g(R)+\bar{e})<0$.

Then problem (1.6) has a solution $u$ such that

$$
r<u<R \text { on }[0, T] \text { and }\left\|u^{\prime}\right\|_{\infty}<R^{\prime} .
$$

Proof. Step 1. For $\lambda \in[0,1]$ and $v \in C_{D}^{1}[0, T]$ denote

$$
\left\{\begin{aligned}
\Xi_{\lambda}(v) & =\int_{0}^{T} g(v(s)) \mathrm{d} s+T \bar{e} \\
& +\sum_{i=1}^{m} M_{i}\left(v, v^{\prime}\right)+\lambda c \sum_{i=1}^{m} J_{i}\left(v, v^{\prime}\right) .
\end{aligned}\right.
$$

Notice that

$$
\Xi_{\lambda}(v)=0 \text { holds for all solutions } v \in C_{D}^{1}[0, T] \text { of (3.1)-(3.4). }
$$

Indeed, let $v \in C_{D}^{1}[0, T]$ be a solution to (3.1)-(3.4). Then

$$
\begin{aligned}
\int_{0}^{T} v^{\prime \prime}(s) \mathrm{d} s & =\sum_{i=0}^{m} \int_{t_{i}}^{t_{i+1}} v^{\prime \prime}(s) \mathrm{d} s=\sum_{i=0}^{m}\left[v^{\prime}\left(t_{i+1}\right)-v^{\prime}\left(t_{i}+\right)\right] \\
& =v^{\prime}(T)-v^{\prime}(0)-\sum_{i=1}^{m} \Delta^{+} v^{\prime}\left(t_{i}\right)=-\lambda \sum_{i=1}^{m} M_{i}\left(v, v^{\prime}\right)
\end{aligned}
$$

and

$$
\begin{aligned}
\int_{0}^{T} c v^{\prime}(s) \mathrm{d} s & =c \sum_{i=0}^{m} \int_{t_{i}}^{t_{i+1}} v^{\prime}(s) \mathrm{d} s=c \sum_{i=0}^{m}\left[v\left(t_{i+1}\right)-v\left(t_{i}+\right)\right] \\
& =c\left[v(T)-v(0)-\sum_{i=1}^{m} \Delta^{+} v\left(t_{i}\right)\right]=-\lambda c \sum_{i=1}^{m} J_{i}\left(v, v^{\prime}\right) .
\end{aligned}
$$


Thus, integrating (3.1) over $[0, T]$ gives (3.6).

Step 2. Consider system (3.7), (3.2), (3.4), where (3.7) is the functionaldifferential equation

$$
v^{\prime \prime}=\lambda\left[-c v^{\prime}+g(v)+e(t)\right]+(1-\lambda) \frac{1}{T} \Xi_{\lambda}(v) .
$$

Due to (3.6), we can see that for each $\lambda \in[0,1]$ the problems (3.1)-(3.4) and (3.7), (3.2)-(3.4) are equivalent. Moreover, for $\lambda=1$, problem (3.7), (3.2), (3.4) reduces to the given problem (1.6) (with $u$ replaced by $v$ ).

Now, notice that in view of (2.21) we have

$$
\int_{0}^{T} G(t, s) \mathrm{d} s=\frac{1}{2} t(t-T) \quad \text { for } t \in[0, T]
$$

and define for $\lambda \in[0,1], \quad u \in C_{D}^{1}[0, T], \quad u>0$ on $[0, T]$, and $t \in[0, T]$

$$
\left\{\begin{array}{l}
\left(F_{\lambda} u\right)(t)=u(0)+u^{\prime}(0)-u^{\prime}(T) \\
+\lambda \int_{0}^{T} G(t, s)\left[-c u^{\prime}(s)+g(u(s))+e(s)\right] \mathrm{d} s \\
+(1-\lambda) \frac{t(t-T)}{2 T} \Xi_{\lambda}(u) \\
\quad-\lambda \sum_{i=1}^{m} \frac{\partial}{\partial s} G\left(t, t_{i}\right) J_{i}\left(u, u^{\prime}\right)+\lambda \sum_{i=1}^{m} G\left(t, t_{i}\right) M_{i}\left(u, u^{\prime}\right) .
\end{array}\right.
$$

In particular, if $\lambda=0$, then

$$
\left(F_{0} u\right)(t)=u(0)+u^{\prime}(0)-u^{\prime}(T)+\frac{t(t-T)}{2 T} \Xi_{0}(u) \text { for } t \in[0, T] .
$$

Let us put

$$
\Omega=\left\{u \in C_{D}^{1}[0, T]: r<u<R \text { on }[0, T] \text { and }\left\|u^{\prime}\right\|_{\infty}<R^{\prime}\right\} .
$$

Arguing similarly to the regular case (see Corollary 2.2), we can conclude that for each $\lambda \in[0,1]$ the operator $F_{\lambda}: \bar{\Omega} \subset C_{D}^{1}[0, T] \rightarrow C_{D}^{1}[0, T]$ is completely continuous and a function $v \in \bar{\Omega}$ is a solution of (3.7), (3.2)-(3.4) if and only if it is a fixed point of $F_{\lambda}$. In particular,

$$
u \in \bar{\Omega} \text { is a solution to (1.6) if and only if } F_{1}(u)=u \text {. }
$$


STEP 3. We will show that

$$
F_{\lambda}(u) \neq u \text { for all } u \in \partial \Omega \text { and } \lambda \in[0,1] .
$$

Indeed, for $\lambda \in(0,1]$ relation (3.10) follows immediately from assumption (i), while for $\lambda=0$ it is a corollary of assumption (ii) and of the following claim.

Claim. $u \in \bar{\Omega}$ is a fixed point of $F_{0}$ if and only if there is $x \in \mathbb{R}$ such that $u(t) \equiv x$ on $[0, T], \quad x \in(r, R)$ and

$$
g(x)+\bar{e}=0 .
$$

Proof of Claim. Let $u \in \bar{\Omega}$ be a fixed point of $F_{0}$, i.e.

$$
u(t)=u(0)+u^{\prime}(0)-u^{\prime}(T)+\frac{t(t-T)}{2 T} \Xi_{0}(u) \text { for all } t \in[0, T] .
$$

Inserting $t=0$ into (3.12), we get $u(0)=u(0)+u^{\prime}(0)-u^{\prime}(T)$, which implies that $u^{\prime}(0)=u^{\prime}(T)$. Similarly, inserting $t=T$ we get $u(T)=u(0)$. Furthermore,

$$
u^{\prime}(t)=\frac{2 t-T}{2 T} \Xi_{0}(u) \text { for } t \in[0, T] .
$$

Since $u^{\prime}(0)=u^{\prime}(T)$, it follows that $\Xi_{0}(u)=0$. This means that $u$ is constant on $[0, T]$. Denote $x=u(0)$. Then $0=\Xi_{0}(u)=T(g(x)+\bar{e})$, i.e., (3.11) is true. On the other hand, it is easy to see that if $x \in \mathbb{R}$ is such that (3.11) holds and $u(t) \equiv x$ on $[0, T]$, then $u \in \bar{\Omega}$ is a fixed point of $F_{0}$. This completes the proof of CLAIM.

STEP 4. By STEP 3 and by the invariance under homotopy property of the topological degree, we have

$$
\operatorname{deg}\left(I-F_{1}, \Omega\right)=\operatorname{deg}\left(I-F_{0}, \Omega\right) .
$$

STEP 5. Let us denote

$$
\mathbb{X}=\left\{u \in C_{D}^{1}[0, T]: u(t) \equiv u(0) \text { on }[0, T]\right\} \quad \text { and } \quad \Omega_{0}=\Omega \cap \mathbb{X} .
$$

Notice that $\Omega_{0}=\{u \in \mathbb{X}: r<u(0)<R\}$ and $\bar{\Omega}_{0}=\{u \in \mathbb{X}: r \leq u(0)<R\}$. By Claim in Step 3, all fixed points of $F_{0}$ belong to $\Omega_{0}$. Hence, by the excision property of the topological degree we have

$$
\operatorname{deg}\left(I-F_{0}, \Omega\right)=\operatorname{deg}\left(I-F_{0}, \Omega_{0}\right) .
$$


SteP 6. Define

$$
\left\{\begin{aligned}
\left(\widetilde{F}_{\mu} u\right)(t)=u(0)+ & {\left[1-\mu+\frac{\mu}{2} t(t-T)\right](g(u(0)+\bar{e})} \\
& \text { for } t \in[0, T], u \in \bar{\Omega}_{0} \text { and } \mu \in[0,1] .
\end{aligned}\right.
$$

We have

$$
\left(\widetilde{F}_{0} u\right)=u(0)+g(u(0))+\bar{e} \text { and }\left(\widetilde{F}_{1} u\right)=F_{0}(u) \quad \text { for each } u \in \mathbb{X} .
$$

Similarly to $F_{\lambda}$, the operators $\widetilde{F}_{\mu}, \mu \in[0,1]$, are also completely continuous and, by Claim in STEP 3, we have

$$
\left(\widetilde{F}_{1} u\right) \neq u \quad \text { for all } \quad u \in \partial \Omega_{0} .
$$

Let $i$ and $i_{-1}$ be respectively the natural isometrical isomorphism $\mathbb{R} \rightarrow \mathbb{X}$ and its inverse, i.e.

$$
i(x)(t) \equiv u \text { for } x \in \mathbb{R} \quad \text { and } \quad i_{-1}(u)=u(0) \text { for } u \in \mathbb{X}
$$

and assume that $\mu \in[0,1), x \in(0, \infty), u=i(x)$ and $\widetilde{F}_{\mu}(u)=u$. Then

$$
\left[1-\mu+\frac{\mu}{2} t(T-t)\right](g(x)+\bar{e})=0 \quad \text { for all } \quad t \in[0, T]
$$

If $t=0$, this relation reduces to $g(x)+\bar{e}=0$, which is due to assumption (ii) possible only if $x \in(r, R)$. To summarize, we have

$$
\left(\widetilde{F}_{\mu} u\right) \neq u \quad \text { for all } u \in \partial \Omega_{0} \text { and all } \mu \in[0,1] .
$$

Hence, using the invariance under homotopy property of the topological degree and taking into account that $\operatorname{dim} \mathbb{X}=1$, we conclude that

$$
\operatorname{deg}\left(I-\widetilde{F}_{1}, \Omega_{0}\right)=\operatorname{deg}\left(I-\widetilde{F}_{1}, \Omega_{0}\right)=\mathrm{d}_{B}\left(I-\widetilde{F}_{0}, \Omega_{0}\right),
$$

where $\mathrm{d}_{B}\left(I-\widetilde{F}_{0}, \Omega_{0}\right)$ stands for the Brouwer degree of $I-\widetilde{F}_{0}$ with respect to the set $\Omega_{0}$ (and the point 0 ).

SteP 7. Define $\Phi: x \in(0, \infty) \rightarrow g(x)+\bar{e} \in \mathbb{R}$. Then

$$
\left(I-\widetilde{F}_{0}\right)(i(x))=i(\Phi(x)) \text { for each } x \in(0, \infty) .
$$


In other words, $\Phi=i_{-1} \circ\left(I-\widetilde{F}_{0}\right) \circ i$ on $(0, \infty)$. Consequently,

$$
\mathrm{d}_{B}\left(I-\widetilde{F}_{0}, \Omega_{0}\right)=\mathrm{d}_{B}(\Phi,(r, R)) .
$$

Now, put

$$
\Psi(x)=\Phi(r) \frac{R-x}{R-r}+\Phi(R) \frac{x-r}{R-r} .
$$

We can see that $\Psi$ has a unique zero $x_{0} \in(r, R)$ and

$$
\Psi^{\prime}\left(x_{0}\right)=\frac{\Phi(R)-\Phi(r)}{R-r} .
$$

Hence, by the definition of the Brouwer degree in $\mathbb{R}$ we have

$$
\mathrm{d}_{B}(\Psi,(r, R))=\operatorname{sign} \Psi^{\prime}\left(x_{0}\right)=\operatorname{sign}(\Phi(R)-\Phi(r)) .
$$

By the homotopy property and thanks to our assumption (iii), we conclude that

$$
\mathrm{d}_{B}(\Phi,(r, R))=\mathrm{d}_{B}(\Psi,(r, R))=\operatorname{sign}(\Phi(R)-\Phi(r)) \neq 0 .
$$

STEP 8. To summarize, by (3.13)-(3.18) we have

$$
\operatorname{deg}\left(I-F_{1}, \Omega\right) \neq 0
$$

which, in view of the existence property of the topological degree, shows that $F_{1}$ has a fixed point $u \in \Omega$. By STEP 1 this means that problem (1.6) has a solution.

\section{References}

[1] Bai Chuanzhi and YAng Dandan. Existence of solutions for second order nonlinear impulsive differential equations with periodic boundary value conditions. Boundary Value Problems, to appear.

[2] D. Bainov And P. Simeonov. Impulsive Differential Equations: Periodic Solutions and Applications. Longman Sci. Tech., Harlow, 1993.

[3] A. Cabada, J. J. Nieto, D. Franco and S. I. Trofimchuk. A generalization of the monotone method for second order periodic boundary value problem with impulses at fixed points. Dynam. Contin. Discrete Impuls. Systems 7 (2000), 145158. 
[4] Dong Yujun Periodic solutions for second order impulsive differential systems. Nonlinear Anal. 27 (1996), 811-820.

[5] L. H. ERBE AND LiU Xinzhi. Existence results for boundary value problems of second order impulsive differential equations. J. Math. Anal. Appl. 149 (1990), 56-69.

[6] Ch.S. HönIG. Volterra Stieltjes-Integral Equations. North Holland and American Elsevier, Mathematics Studies 16, Amsterdam and New York, 1975.

[7] Hu Shouchuan and V. Laksmikantham. Periodic boundary value problems for second order impulsive differential systems. Nonlinear Anal. 13 (1989), 75-85.

[8] LeE Yong-Hoon And LiU Xinzhi. Study of singular boundary value problem for second order impulsive differential equations. J, Math. Anal. Appl. 331 (2007), 159176.

[9] E. Liz AND J. J. Nieto. Periodic solutions of discontinuous impulsive differential systems. J. Math. Anal. Appl. 161 (1991), 388-394.

[10] E. Liz AND J. J. Nieto. The monotone iterative technique for periodic boundary value problems of second order impulsive differential equations. Comment. Math. Univ. Carolin. 34 (1993), 405-411.

[11] R. Manásevich and J. Mawhin. Periodic solutions for nonlinear systems with p-Laplacian-like operators. J. Differential Equations 145 (1998), 367-393.

[12] J. Mawhin. Topological degree methods in nonlinear boundary value problems. In: Regional Conference Series in Mathematics. No.40. R.I.: The American Mathematical Society (AMS). 1979, 122 p.

[13] J. Mawhin. Topological Degree and Boundary Value Problems for Nonlinear Differential Equations. In: Topological methods for ordinary differential equations. (M. Furi and P. Zecca, eds.) Lect. Notes Math. 1537, Springer, Berlin, 1993, pp. 73-142.

[14] I. RACHŮnková. Singular Dirichlet second order boundary value problems with impulses. J. Differential Equations 193 (2003), 435-459.

[15] I. Rachůnková, S. StanĚK And M. TVRdÝ. Singularities and Laplacians in Boundary Value Problems for Nonlinear Ordinary Differential Equations. In: Handbook of Differential Equations. Ordinary Differential Equations, vol.3. (A. Caňada, P. Drábek, A. Fonda, eds.) Elsevier 2006, pp. 607-723.

[16] I. Rachůnková, S. StanĚK And M. TvRdÝ. Solvability of Nonlinear Singular Problems for Ordinary Differential Equations. Hindawi [Contemporary Mathematics and Its Applications, Vol.5], in print.

[17] I. RACHŮnková AND J. TOMEČEK. Singular Dirichlet problem for ordinary differential equations with impulses. Nonlinear Anal., Theory Methods Appl. 65 (2006), 210-229.

[18] I. RachŮnková And M. TvRdÝ. Impulsive Periodic Boundary Value Problem and Topological Degree. Funct. Differ. Equ. 9, no.3-4, 471-498.

[19] I. RACHŮnKová AND M. TVRDÝ. Nonmonotone impulse effects in second order periodic boundary value problems. Abstr. Anal. Appl. 2004: 7, 577-590.

[20] I. RACHŮnková AND M. TVRDÝ. Non-ordered lower and upper functions in second order impulsive periodic problems. Dyn. Contin. Discrete Impuls. Syst., Ser. A, Math. Anal. 12 (2005), 397-415. 
[21] I. RACHŮNKOVÁ AND M. TVRDÝ. Existence results for impulsive second order periodic problems, Nonlinear Anal., Theory Methods Appl. 59 (2004) 133-146.

[22] I. RAChŮNKová AND M. TvRDÝ. Method of lower and upper functions in impulsive periodic boundary value problems. In: EQUADIFF 2003. Proceedings of the International Conference on Differential Equations, Hasselt, Belgium, July 22-26,2003, ed. by F. Dumortier, H. Broer, J. Mawhin, A. Vanderbauwhede, S. Verduyn, Hackensack, NJ, World Scientific (2005), pp. 252-257.

[23] I. Rachưnková And M. TvRdÝ. Second Order Periodic Problem with $\phi$-Laplacian and Impulses - Part I. Mathematical Institute of the Academy of Sciences of the Czech Republic, Preprint 155/2004 [available as \http://www.math.cas.cz/ tvrdy/lapl1.pdf or \http://www.math.cas.cz/ tvrdy/lapl1.ps].

[24] I. RachŮnková And M. TVRdÝ. Second Order Periodic Problem with $\phi$-Laplacian and Impulses - Part II. Mathematical Institute of the Academy of Sciences of the Czech Republic, Preprint 156/2004 [available as \http: //www.math.cas.cz/ tvrdy/lap12.pdf or \http://www.math.cas.cz/ tvrdy/lapl2.ps].

[25] I. RACHŮnKová AND M. TVRDÝ. Second order periodic problem with phi-Laplacian and impulses. Nonlinear Analysis, T.M.A. 63 (2005), e257-e266.

[26] I. Rachůnková and M. TvrdÝ. Periodic singular problem with quasilinear differential operator. Mathematica Bohemica 131 (2006), 321-336.

[27] Š. SChWABIK AND M. TVRDÝ. Boundary value problems for generalized linear differential equations, Czechoslovak Math. J. 29 (104) (1979), 451-477.

[28] Š. Schwabik, M. Tvrdý And O. Vejvoda. Differential and Integral Equations: Boundary Value Problems and Adjoint. Academia and D. Reidel, Praha and Dordrecht, 1979.

[29] M. TVRDÝ. Fredholm-Stieltjes integral equations with linear constraints: duality theory and Green's function. Časopis pěst. mat. 104 (1979), 357-369.

[30] Zhang Zhitao Existence of solutions for second order impulsive differential equations. Appl. Math., Ser. B (Engl. Ed.) 12 (1997), 307-320. 